\title{
Iterative Improvement of Path Accuracy of Industrial Robots Using External Measurements
}

\author{
Peter Stückelmaier, Martin Grotjahn and Carsten Fräger
}

\begin{abstract}
This paper presents a cascaded methodology for enhancing the path accuracy of industrial robots by using advanced control schemes. It includes kinematic calibration as well as dynamic modeling and identification. This is followed by a centralized model-based compensation of robot dynamics. The implemented feed-forward torque control shows the expected improvements of control accuracy. However, external measurements show the influence of joint elasticities as systematic path errors. To further increase the accuracy an iterative learning controller (ILC) based on external camera measurements is designed. The implementation yields to significant improvements of path accuracy. By means of a kind of automated "Teach-In", an overall effective concept for the automated calibration and optimization of the accuracy of industrial robots in high-dynamic path-applications is realized.
\end{abstract}

\section{INTRODUCTION}

Despite of stiff construction, industrial robots with serial kinematics are excited to oscillate in high-dynamic applications, primarily because of gearbox elasticity and backlash. This leads to minor path accuracy. To reduce this negative effect, model-based control methods can be used. For this purpose, these influences must be measured and quantitatively analyzed on the arm-side.

The internal robot joint measurement (motor angle) can not exactly represent the real path. One possibility for precise measuring of high-dynamic trajectories are powerful 3Dcamera measurement systems, which allows high-frequency scanning of the Tool-Center-Point (TCP) path. This results in a detailed depiction of the actual path and reveals the oscillation of the robots arm. Fig. 1 shows the high difference to motorside measurements.
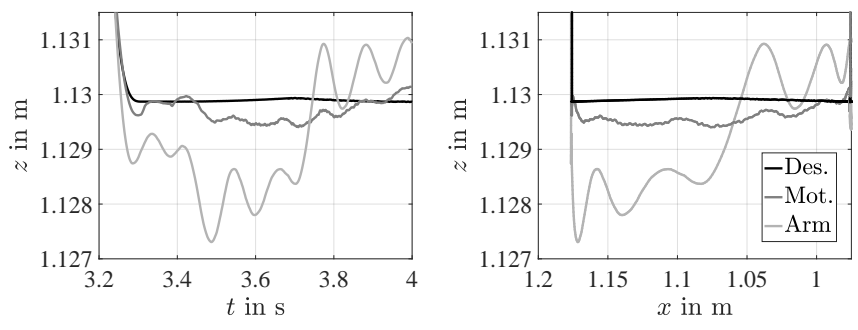

Fig. 1. Path accuracy using motor-side and arm-side measurements

Peter Stückelmaier, Martin Grotjahn and Carsten Fräger are with the Institut für Konstruktionselemente, Mechatronik und Elektromobilität (IKME), University of Applied Sciences, Hannover, Germany. (phone: +49 $5119296-$ 1345; e-mail: \{peter.stueckelmaier, martin.grotjahn, carsten.fraeger\}@hshannover.de).
This paper presents a cascaded methodology for significant improvement of the arm-side path accuracy of serial robots fast path applications. The methodology consists of three steps:

1. kinematic calibration as well as dynamic modeling and identification,

2. compensation of the non-linear coupled multi-body dynamics using model-based feed-forward torque control and

3. reduction of remaining path errors using iterative learning control (ILC) based on external measurements.

A calibration of the camera and the robot kinematics is essential for the high-dynamic measurement of the TCPposition in task space. This applies to model-based approaches, whose parameters are experimentally identified.

Another prerequisite is the inverse dynamic model of the serial robot. It can be derived by using classical mechanics (e.g. [1]). The correct values of the involved parameters are not always available. Especially, those dealing with friction and compliance characteristics are completely unknown. Thus, experimental robot identification is the only efficient way to obtain accurate models of robot dynamics.

The experimental design, which includes optimization of excitation trajectories, is fundamental for effective identification of the model parameters. In Swevers et al. [2] for each joint a Fourier series approach is utilized as desired trajectory. This approach has proven in our own [3] and others investigations [4]-[7] as very robust and reliable. It is also adopted in this work.

The designed experiments and their influence on identification and model accuracy is tested for a KUKA KR 6-2 industrial robot. The maximum payload of $6 \mathrm{~kg}$ is applied to the robot to perform the experiments under realistic conditions. A developed test bench with the restriction-free possibility to implement advanced control concepts is available for the technical realization. The model-based feed-forward torque control based on a coupled multi-body model of the inverse dynamics [3] leads to a significant reduction in tracking errors on the motor-side. Nevertheless, unmodeled effects (such as joint elasticities) and disturbances lead to high path deviations of the TCP.

Further accuracy improvement are achieved by an iteratively learning control (ILC) algorithm based on external camera measurements. This can iteratively compensate unknown systematic disturbances by corrections of the desired trajectory. Thus, it leads to a considerable increase in accuracy. Because of numerical stability and robustness, linear time-discrete models are often used to describe the system behavior [7]-[11]. 


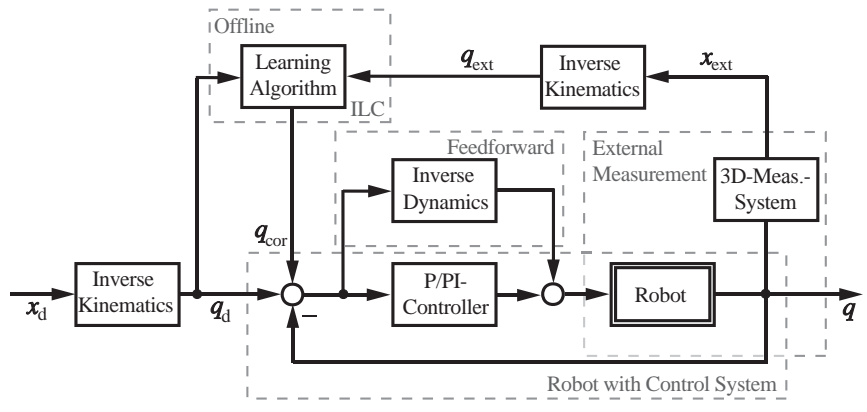

Fig. 2. Schematic representation of the proposed cascaded methodology

The approach chosen here is based on the model presented in Lange and Hirzinger [12] and further developed in [13], [14]. The capacity of the approach has been successfully presented in [15] for learning of motor-side position.

To increase the arm-side path accuracy effectively, in this work the learning is based on external measurements of the TCP trajectory. For this purpose, the Cartesian path deviations of the end-effector are transformed into the joint angles by inverse kinematics. By means of a kind of automated "TeachIn", an overall effective concept for the automated calibration and optimization of the accuracy of industrial robots in highdynamic path-applications is realized.

\section{Methodology For InCREASING THE ACCURACY}

This section describes the single steps of the presented methodology to increase the path accuracy of serial robots using external measurements. A schematic representation of the overall concept is shown in Fig.2.

\section{A. Modeling and identification}

1) Kinematics: The KUKA KR 6-2 is a serial robot with 6 rotational joints. For linear estimation of the dynamics, the use of the modified DENAVIT-HARTENBERG (MDH) convention [16] is advantageous [17], [18]. Fig. 3 shows the defined coordinate systems and Tab. [1] shows the MDH-parameters.

A kinematic calibrated robot system is the basis to investigate and optimize the path accuracy. The calibration of the robot kinematics is carried out by using the $6 \mathrm{D}$ calibration presented by Wiest [19]. The kinematic errors are compensated by a model-based approach where the MDH-parameters from Tab.I are experimentally identified.

TABLE I

THE MDH-PARAMETERS of the KUKA KR 6-2 $l_{0}=0.675 \mathrm{~m}, l_{1}=0.26 \mathrm{~m}, l_{2}=0.68 \mathrm{~m}, l_{3}=0.035 \mathrm{~m}, l_{4}=0.67 \mathrm{~m}, l_{\mathrm{E}}=0.115 \mathrm{~m}$

\begin{tabular}{|c|c|c|c|c|}
\hline Joint $i$. & $\theta_{i}$ in $\mathrm{rad}$ & $d_{i}$ in $\mathrm{m}$ & $a_{i}$ in $\mathrm{m}$ & $\alpha_{i}$ in $\mathrm{rad}$ \\
\hline 1 & $-q_{1}$ & $l_{0}$ & 0 & 0 \\
2 & $q_{2}$ & 0 & $l_{1}$ & $-\frac{\pi}{2}$ \\
3 & $q_{3}-\frac{\pi}{2}$ & 0 & $l_{2}$ & 0 \\
4 & $-q_{4}$ & $l_{4}$ & $-l_{3}$ & $-\frac{\pi}{2}$ \\
5 & $q_{5}$ & 0 & 0 & $\frac{\pi}{2}$ \\
6 & $-q_{6}+\pi$ & $l_{\mathrm{E}}$ & 0 & $-\frac{\pi}{2}$ \\
\hline
\end{tabular}

2) Dynamics: As part of the modeling only rigid-body and friction dynamics are taken into account.

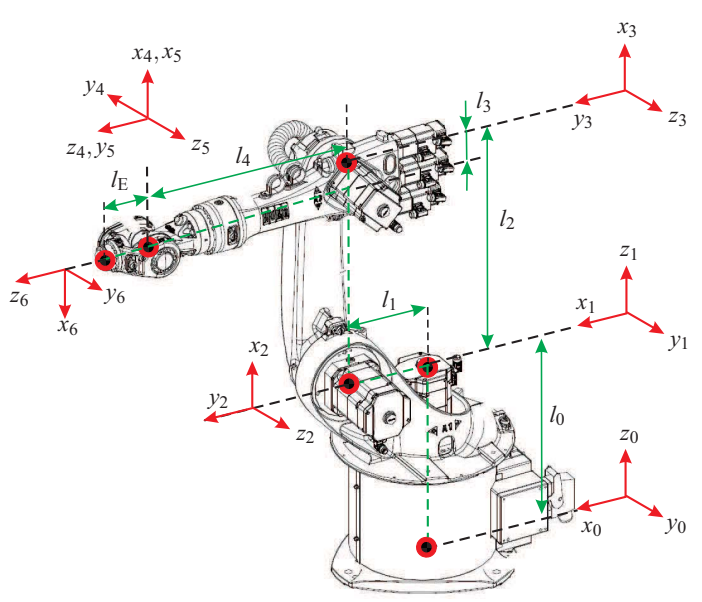

Fig. 3. Kinematics of the KUKA KR 6-2 defined by the MDH-convention $\left(q_{2}=-\frac{\pi}{2}\right.$ and $\left.q_{3}=\frac{\pi}{2}\right)$

Rigid-body model: The rigid-body model is considered as a set of $n$ rigid bodies interconnected by revolute joints. The rigid-body dynamics can be analytically calculated in parameter-linear form by use of Jourdain's principle [7], [15]:

$$
\begin{gathered}
\boldsymbol{\tau}=\sum_{i=1}^{n} \underbrace{\left[\boldsymbol{J}_{\mathrm{T}_{i}}^{\mathrm{T}}\left(m_{i(i)} \dot{\boldsymbol{v}}_{i}+{ }_{(i)} \dot{\tilde{\boldsymbol{\omega}}}_{i} \boldsymbol{s}_{i}+{ }_{(i)} \tilde{\boldsymbol{\omega}}_{i(i)} \tilde{\boldsymbol{\omega}}_{i} \boldsymbol{s}_{i}\right)\right]}_{\text {translatory part }}+ \\
+\sum_{i=1}^{n} \underbrace{\left[\boldsymbol{J}_{\mathrm{R}_{i}}^{\mathrm{T}}\left({ }_{(i)} \boldsymbol{I}_{i(i)} \dot{\boldsymbol{\omega}}_{i}+{ }_{(i)} \tilde{\boldsymbol{\omega}}_{i}\left({ }_{(i)} \boldsymbol{I}_{i(i)} \boldsymbol{\omega}_{i}\right)+\tilde{\boldsymbol{s}}_{i(i)} \dot{\boldsymbol{v}}_{i}\right)\right]}_{\text {rotatory part }},
\end{gathered}
$$

with the inertia tensor ${ }_{(i)} \boldsymbol{I}_{i}$, first-order moment $\boldsymbol{s}_{i}$, translational and angular velocities ${ }_{(i)} \boldsymbol{v}_{i},{ }_{(i)} \boldsymbol{\omega}_{i}$ and accelerations ${ }_{(i)} \dot{\boldsymbol{v}}_{i},{ }_{(i)} \dot{\boldsymbol{\omega}}_{i}$ of link $i$ in frame $i$ (see Fig. 3). $\boldsymbol{J}_{\mathrm{T}_{i}}$ and $\boldsymbol{J}_{\mathrm{R}_{i}}$ are the Jacobians and $\tau$ represents the vector of drive torques.

Thus, the equations of motion (1) can be rewritten in parameter-linear matrix notation [15]:

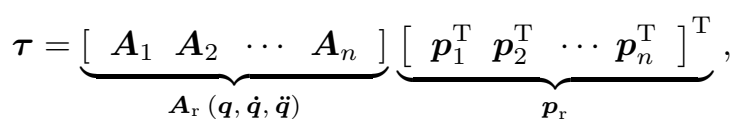

with the matrix $\boldsymbol{A}_{i}$ as a function of kinematic parameters as well as the joint angles, velocities and accelerations. The parameter vector $\boldsymbol{p}_{i}$ of the $i$-th link consists of the parameters $\left[I_{i_{\mathrm{xx}}}, I_{i_{\mathrm{xy}}}, I_{i_{\mathrm{xz}}}, I_{i_{\mathrm{yy}}}, I_{i_{\mathrm{yz}}}, I_{i_{\mathrm{zz}}}, s_{i_{\mathrm{xx}}}, s_{i_{\mathrm{yy}}}, s_{i_{\mathrm{zz}}}, m_{i}\right]$. Thus, the overall parameter vector $\boldsymbol{p}_{\mathrm{r}}$ of the $n$ joints has $10 n$ elements.

Friction: Although friction behaviour is complex, a simple model is often used to describe the joint torques [2], [5], [7], [15:

$$
\tau_{\mathrm{f}, i}=\underbrace{F_{\mathrm{c}, i} \operatorname{sgn}\left(\dot{q}_{i}\right)}_{\text {Coulomb }}+\underbrace{F_{\mathrm{v}, i} \dot{q}_{i}}_{\text {viscous }},
$$

with the Coulomb and viscous friction coefficients $F_{\mathrm{c}, i}$ and $F_{\mathrm{v}, i}$. This modeled characteristics is valid for the steady-state condition, which is reached after half an hour of continuous operation (quasi-constant motor temperature; uniform lubricant distribution in the gearbox). The parameter-linear matrix 
notation is shown in (4) where $\boldsymbol{p}_{\mathrm{f}}$ contains the $2 n$ friction coefficients of the $n$ joints:

$$
\boldsymbol{\tau}_{\mathrm{f}}=\boldsymbol{A}_{\mathrm{f}}(\dot{\boldsymbol{q}}) \boldsymbol{p}_{\mathrm{f}} .
$$

Base parameters: One prerequisite for linear parameter estimation is a parameter vector of minimum dimension. Some parameters of the rigid-body model cannot be identified because they have no effect on the dynamics. Some parameters' influences linearly depend on some others'. The implemented reduction procedure is based on the regrouping rules defined in [15], [17], [18]. The corresponding columns of the matrix $\boldsymbol{A}_{\mathrm{r}}$ are removed, so that a regular matrix arises. The resulting identifiable parameters are called base parameters.

The overall system equation results from the combination of the minimal parametrized rigid-body model with the friction model:

$$
\boldsymbol{\tau}=\boldsymbol{A}_{\Sigma}(\boldsymbol{q}, \dot{\boldsymbol{q}}, \ddot{\boldsymbol{q}}) \boldsymbol{p}_{\Sigma}
$$

The resulting base parameter vector $\left(\operatorname{dim}\left(\boldsymbol{p}_{\Sigma}\right)=29\right)$ can be found in [3].

3) Experimental identification of robot dynamics: The methodology is based on (5). It allows the use of computationally efficient linear estimation methods [2], [4], [7], [15]. The aim is to identify all parameters of the dynamic model in an unbiased and robust way by using only one appropriate measurement process. The estimation problem can be formulated as follows:

$$
\underbrace{\left[\begin{array}{c}
\tilde{\boldsymbol{\tau}}\left(t_{1}\right) \\
\tilde{\boldsymbol{\tau}}\left(t_{2}\right) \\
\vdots \\
\tilde{\boldsymbol{\tau}}\left(t_{N}\right)
\end{array}\right]}_{\boldsymbol{\Xi}}=\underbrace{\left[\begin{array}{c}
\boldsymbol{A}_{\Sigma}\left(\boldsymbol{\xi}\left(t_{1}\right)\right) \\
\boldsymbol{A}_{\Sigma}\left(\boldsymbol{\xi}\left(t_{2}\right)\right) \\
\vdots \\
\boldsymbol{A}_{\Sigma}\left(\boldsymbol{\xi}\left(t_{N}\right)\right)
\end{array}\right]}_{\boldsymbol{\Psi}} \boldsymbol{p}_{\Sigma}+\underbrace{\left[\begin{array}{c}
\boldsymbol{e}\left(t_{1}\right) \\
\boldsymbol{e}\left(t_{2}\right) \\
\vdots \\
\boldsymbol{e}\left(t_{N}\right)
\end{array}\right]}_{\boldsymbol{\epsilon}},
$$

where the vector $\boldsymbol{\xi}\left(t_{i}\right)$ defines the kinematic measurements at time $t_{i}, \boldsymbol{\Psi}$ denotes the full-rank observation matrix computed for the $N$ measurement points and $\boldsymbol{\Xi}$ is the vector obtained from the $N$ measured torque vectors $\tilde{\boldsymbol{\tau}}\left(t_{i}\right) . \boldsymbol{\epsilon}$ is the vector of measurement noise.

Since errors mainly occur in the torque measurement $\tilde{\tau}$ the consideration of purely additive disturbances is proposed. Thus, $\epsilon$ is assumed as an additive zero-mean, Gaussian white noise vector $\left(E\left(\boldsymbol{\epsilon} \boldsymbol{\epsilon}^{\mathrm{T}}\right)=\sigma^{2} \boldsymbol{E}\right)$. Furthermore, if $\boldsymbol{\Psi}$ is assumed as deterministic and error-free, the Least-Square approach is the unbiased estimator of minimal variance. Due to simplicity of linear estimators, most of the published schemes in robotics solve (6) using Least-Square methods [3], [7], [15].

$$
\begin{aligned}
\hat{\boldsymbol{p}}_{\Sigma} & =\arg \left\{\min _{\boldsymbol{p}}\left(\left(\boldsymbol{\Psi} \boldsymbol{p}_{\Sigma}-\boldsymbol{\Xi}\right)^{\mathrm{T}}\left(\boldsymbol{\Psi} \boldsymbol{p}_{\Sigma}-\boldsymbol{\Xi}\right)\right)\right\} \\
& =\left(\boldsymbol{\Psi}^{\mathrm{T}} \boldsymbol{\Psi}\right)^{-1} \boldsymbol{\Psi}^{\mathrm{T}} \boldsymbol{\Xi} .
\end{aligned}
$$

The necessary deterministic observations must be made in a suitable measuring process. So, the information content of $\Psi$ and the excitation of the system must be maximized. The approach of Swevers et al. [2], which arrange a Fourier series for each joint as excitation trajectory, combined with an optimization of the path parameters by minimizing the determinant of $\boldsymbol{\Psi}$ (D-optimality) [20] leads to high excitation. Furthermore, due to the possibility of analytical reconstruction of the path parameters, almost noise-free deterministic measurements are available. Thus, an unbiased parameter estimation of $\hat{\boldsymbol{p}}_{\Sigma}$ is to be expected. This has been successfully implemented and proven experimentally on various robotic systems [4]-[7].

\section{B. Model-based feed-forward torque control}

With the identified parameters $\hat{p}_{\Sigma}$ and the analytic model of the inverse dynamics (5), feed-forward torques can be calculated for each drive. Thus, a model-based feed-forward torque control enables effective compensation of non-linear coupled multi-body dynamics. This successfully reduces the motor-side tracking errors [3]. It is integrated into the advanced control scheme of the cascaded methodology to improve the arm-side path accuracy in high-dynamic robot trajectories (see Fig.22.

\section{Iterative Learning Control (ILC)}

Due to simplified modeling and unconsidered effects, model-based control concepts can not eliminate all path errors. This leads to remaining path deviations. An iterative learning control algorithm (ILC) can compensate systematic errors. Thus, the accuracy of the complex nonlinear and coupled systems will be significantly increased. Because of simple applicability and high robustness against disturbances and model errors, linear time-invariant model-based approaches [7]-[11] are often used. Based on measured path deviations, the path accuracy is increased iteratively:

$$
\boldsymbol{q}_{\mathrm{d}, \mathrm{cor}}^{p}=\boldsymbol{q}_{\mathrm{d}, \mathrm{cor}}^{p-1}+\boldsymbol{L}\left(\boldsymbol{q}_{\mathrm{d}}, \boldsymbol{q}^{p-1}\right)
$$

with the corrected desired joint angles of the actual $\boldsymbol{q}_{\mathrm{d}, \mathrm{cor}}^{p}$ and previous $\boldsymbol{q}_{\mathrm{d}, \mathrm{cor}}^{p-1}$ learning iteration and the learning function $\boldsymbol{L}$ which consists of the nominal desired joint angles $\boldsymbol{q}_{\mathrm{d}}$ and the actual joint angles $\boldsymbol{q}^{p-1}$ of the previous learning iteration. The performance of the ILC strongly depends on the properties of $L$.

All single-axis control loops are assumed to be linear and decoupled. Due to simple identification 1 and system inversion, time-discrete impulse response models are used. The relationship between the desired joint value $q_{\mathrm{d}}(k)$ and its actual value $q(k)$ can be described as follows:

$$
q(k)=\sum_{l=1}^{m_{\mathrm{g}}} g(l) q_{\mathrm{d}}(k-l+1)+e(k)=\boldsymbol{q}_{\mathrm{d}}(k)^{\mathrm{T}} \boldsymbol{g}+e(k) .
$$

For stable systems, the time-discrete impulse response $\boldsymbol{g}$ is sufficiently accurately described by a limited number of elements $m_{g}$. With the desired joint angles $\boldsymbol{q}_{\mathrm{d}}(k)$ and an additive unknown error $e(k)$ the determination of $\hat{\boldsymbol{g}}$ is realized analogously to (7) using Least-Square identification:

$$
\hat{\boldsymbol{g}}=\left(\boldsymbol{\Psi}_{q_{\mathrm{d}}}^{\mathrm{T}} \boldsymbol{\Psi}_{q_{\mathrm{d}}}\right)^{-1} \boldsymbol{\Psi}_{q_{\mathrm{d}}}^{\mathrm{T}} \boldsymbol{q}
$$

\footnotetext{
${ }^{1}$ The observation matrix $\boldsymbol{\Psi}\left(\boldsymbol{q}_{\mathrm{d}}\right)$ contains only desired values and is therefore always error-free and deterministic. Thus, a basic requirement of the linear estimation is ensured [21].
} 
with $\boldsymbol{\Psi}_{q_{\mathrm{d}}}$ as the observation matrix filled with the vectors of the desired values $\boldsymbol{q}_{\mathrm{d}}$ for $m_{\mathrm{g}}$ time steps from (9) and $\boldsymbol{q}$ is the vector of the actual joint positions.

Experiments reveal that, in the case of high-frequency excitation by dynamic trajectories, the convergence ${ }^{2}$ of the iterative learning is not always guaranteed. Thus, oscillations are often excited. Therefore smoothing of the desired trajectory is proposed. Lange and Hirzinger [14] apply a special inversion method Inverse-Covariance Kalman-Filter. Other authors bypass inversion by directly filtering the corrections by means of a Butterworth filter [9], or completely exclude filtering by the so-called Contraction Mapping [7], [8]. According to [15], an Extended Least-Square (ELS) approach allows similar results with lower complexity and easier parameterization.

The ELS smooths the desired trajectory by regularizing of the poorly conditioned estimation problem. The following linear system of equations results for joint $i$ :

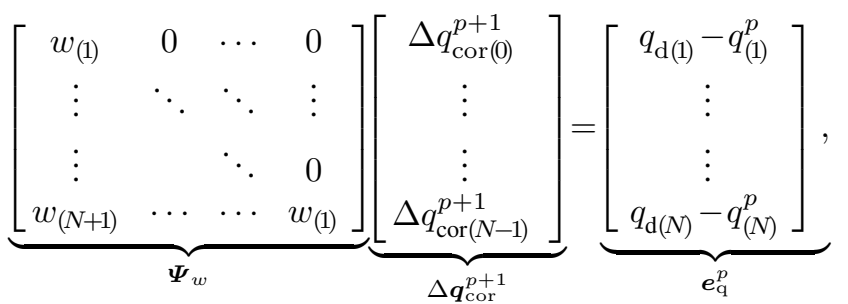

with the step response elements $w(k)=\sum_{j=0}^{k} g(j)$ which can be determined from (10). Analogously to $(6), \boldsymbol{\Psi}_{w}$ is the observation matrix, $\boldsymbol{e}_{\mathrm{q}}^{p}$ is the vector of joint errors and $\Delta \boldsymbol{q}_{\mathrm{cor}}^{p+1}$ is the sought parameter vector. So, instead of absolute corrections the changes of the corrections between two successive time-steps are estimated.

The regularization of the estimates results from a heuristic extension of the ordinary Least-Square cost function according to [20], [21]:

$$
J(\boldsymbol{p})=\frac{1}{2} \boldsymbol{\epsilon}^{\mathrm{T}} \boldsymbol{\epsilon}+\frac{h}{2} \boldsymbol{p}^{\mathrm{T}} \boldsymbol{p} \longrightarrow \min _{\boldsymbol{p}},
$$

with the extended cost function $J$, the error vector $\epsilon$ and the parameter vector $\boldsymbol{p}$. The following solution of (11) results:

$$
\Delta \hat{\boldsymbol{q}}_{\mathrm{cor}}^{p+1}=\left(h \boldsymbol{E}+\boldsymbol{\Psi}_{w}^{\mathrm{T}} \boldsymbol{\Psi}_{w}\right)^{-1} \boldsymbol{\Psi}_{w}^{\mathrm{T}} \boldsymbol{e}_{\mathrm{q}}^{p} .
$$

The regularization parameter $h$ allows to penalize the correction changes $\Delta \boldsymbol{q}_{\text {cor }}$ and leads to a smoothing of the corrected desired path. Advantageous is a lower excitation of oscillation as well as a significantly more robust convergence behavior of the learning process [15].

Consideration of Cartesian path error: According to the schematic representation in Fig.2 the increase of the armside path accuracy is based on external measurements of the Cartesian path deviations of the end-effector $\boldsymbol{e}_{\mathrm{x}}$. However, for the iterative path correction by (13) the learning take place on the basis of joint error measurements $e_{\mathrm{q},(k)}=q_{\mathrm{d}(k)}-q_{(k)}^{*}$. For this purpose, in this work the inverse kinematics according to

\footnotetext{
${ }^{2}$ The requirement for the learning algorithm is its convergence with respect to a true desired trajectory $\lim _{p \rightarrow \infty} \boldsymbol{q}_{\mathrm{d}, \mathrm{por}}^{p}=\boldsymbol{q}_{\mathrm{d}, \mathrm{T}}$.
}

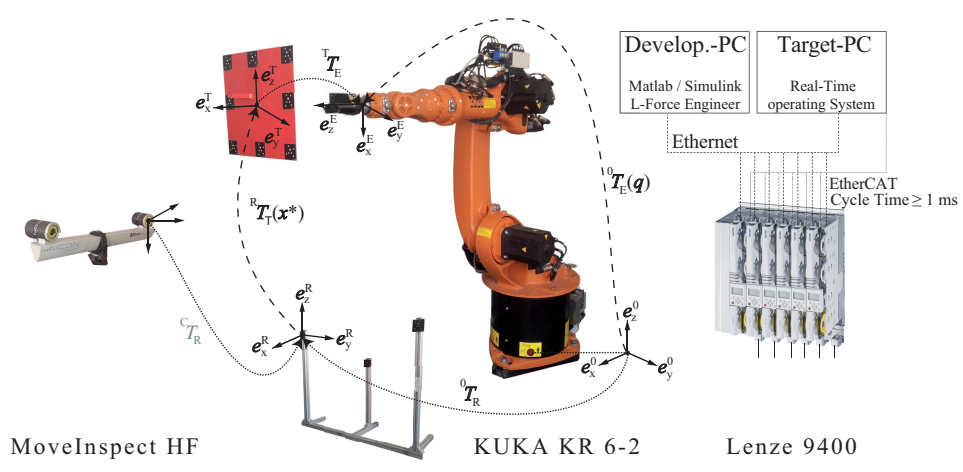

Fig. 4. Test bench for the implementation of the developed control concepts

Paul and Zhang [22] is used to transform the external Cartesian measurements of the end-effector $\boldsymbol{x}^{*}$ to the individual robot axes $\boldsymbol{q}^{*}$.

\section{EXPERIMENTAL METHODOLOGY TESTING}

The capacity of the presented methodology for practical applications is experimentally investigated on a test bench. The improvement of the dynamic path accuracy is evaluated by using the camera measurements of TCP position.

\section{A. Testing environment}

The test bench for the investigation of the arm-side accuracy includes a connection of the servo synchronous motors of a KUKA KR 6-2 industrial robot to the established Lenze 9400 inverters according to Fig. 4 The developed control system is characterized by the following:

- extensive and user-friendly development- and real-timeenvironment (MathWorks Simulink-Real-Time),

- short control cycle time (1 ms),

- computed torque control interface,

- restriction-free application of arbitrary trajectories and

- data-fusion of motor and arm angle measurements.

To examine the arm-side path accuracy Aicons Cartesian 3D measurement system MoveInspect $H F^{3}$ is integrated into the test bench. This measures the position at high frequencies up to $500 \mathrm{~Hz}$ with an accuracy of up to $0.1 \mathrm{~mm}$. The main advantage of the external arm-side measurement is, that all systematic errors (elasticity, backlash, friction, etc.) are collected by measuring the robot end-effector position.

A camera calibration is required for the application of the method. Therefore, the position of the TCP is measured in relation to a fixed reference frame (see Fig.47. Based on this, a coordinate frame with an orthogonal basis is generate in reference coordinates ${ }^{\mathrm{R}} \boldsymbol{T}_{\mathrm{T}}\left(\boldsymbol{x}^{*}\right)$. For calibration, the transformation of the external end-effector measurements $\boldsymbol{x}^{*}$ with respect to the base frame of the robot ${ }^{0} \boldsymbol{T}_{\mathrm{E}}\left(\boldsymbol{x}^{*}\right)$ must be known. For this purpose, two constant transformations are required (see Fig. (4):

$$
{ }^{0} \boldsymbol{T}_{\mathrm{E}}\left(\boldsymbol{x}^{*}\right)={ }^{0} \boldsymbol{T}_{\mathrm{R}}{ }^{\mathrm{R}} \boldsymbol{T}_{\mathrm{T}}\left(\boldsymbol{x}^{*}\right){ }^{\mathrm{T}} \boldsymbol{T}_{\mathrm{E}},
$$

${ }^{3}$ http://aicon3d.com/products/moveinspect-technology/moveinspecthf/technical-details.html 

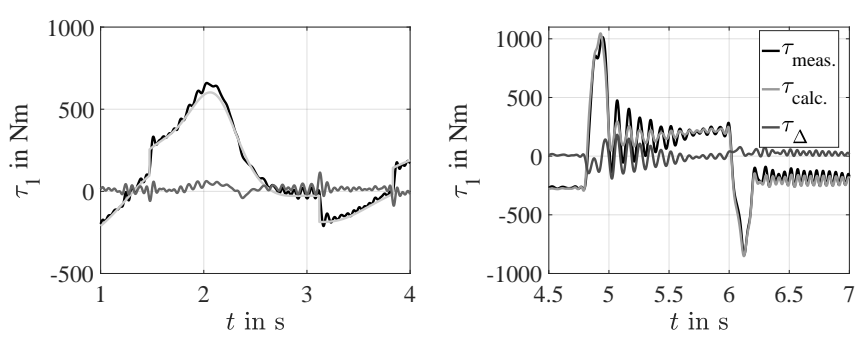

Fig. 5. Comparison between the measured and the calculated torque of joint 1; left: identification trajectory, right: validation trajectory

with the transformation of the reference frame with respect to the base frame of the robot ${ }^{0} \boldsymbol{T}_{\mathrm{R}}$ as well as the transformation of the end-effector with respect to the measured TCP ${ }^{\mathrm{T}} \boldsymbol{T}_{\mathrm{E}}$.

Thus, by means of non-linear optimization, the following target function with respect to the parameters $\boldsymbol{\eta}$ is minimized by comparing the external data $\boldsymbol{x}^{*}$ with the internal robot sensor data $\boldsymbol{q}$ :

$$
\hat{\boldsymbol{\eta}}=\arg \left\{\min _{\hat{\boldsymbol{\eta}}}\left\|^{0} \boldsymbol{T}_{\mathrm{R}}(\hat{\boldsymbol{\eta}}){ }^{\mathrm{R}} \boldsymbol{T}_{\mathrm{T}}\left(\boldsymbol{x}^{*}\right)^{\mathrm{T}} \boldsymbol{T}_{\mathrm{E}}(\hat{\boldsymbol{\eta}})-{ }^{0} \boldsymbol{T}_{\mathrm{E}}(\boldsymbol{q})\right\|^{2}\right\} .
$$

The precisely calibrated 3D measuring system is used in the following to increase the path accuracy of high-dynamic trajectories.

\section{B. Identification and validation}

The identification procedure discussed in Sec.II-A3 is applied to the robot KUKA KR 6-2. The model parameters of robot dynamics are identified using LS estimation (7) considering the minimally parameterized model structure according to (5) and the trajectory design using the Fourier approach (see [2]) and its optimization.

The investigation of the estimation quality is carried out by statistical analysis of relative standard deviations of the estimation error. Due to the optimized excitation, especially all parameters with a high influence on the dynamics (high magnitude) are identified with a small expected estimation error $<5 \%$. The analysis of the standard deviation of the parameter estimates shows similar results. Most parameters with a high influence on the robot's dynamics always remain centered in their confidence interval (for details, see [3]).

For validation, the joint torques are predicted using the identified model according to (5). The results for joint 1 are exemplary depicted in Fig.5 for two trajectories which are different in their dynamic characteristics.

Qualitatively, one can see a good agreement of calculated and measured torque. Especially the comparison for the identification trajectory shows only slight deviations. These occur due to complex friction effects which excite joint elasticity, when the velocity crosses zero. For the validation trajectories, deviations become particularly evident by torque oscillations caused again by excitation of joint elasticity.

\section{Dynamic compensation using feed-forward torque control}

The first control approach for improving the arm-side accuracy is model-based feed-forward torque control. The

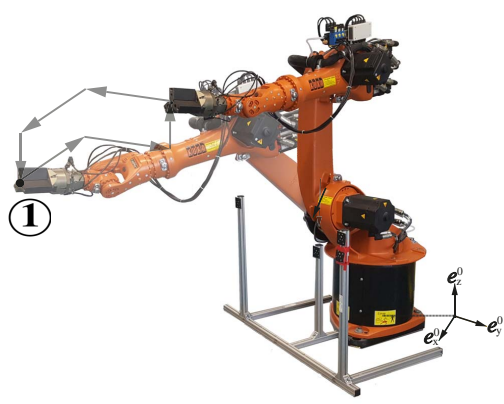

Fig. 6. Linear motion in Cartesian space (Conditions: maximum speed and payload; Configuration (1): maximum outreach while maintaining visibility)

implementation in [3] significantly reduced the motor-side tracking error. In this work the impact on TCP accuracy is examined. The following conceptual control implementations are compared:

- CF: Developed test bench in mode Cascaded feedback PID-type controller,

- MFTC: Developed test bench in mode Cascaded feedback PID-type controller with model-based feedforward torque control.

The investigations are performed using linear motions in Cartesian space generated by the KUKA KR C4 under "worstcase" conditions including maximum speed as well as maximum payload. The use of a camera reduce the workspace, so it must be ensured that the path of the robot satisfies the boundary conditions of visibility. The investigated trajectorie is shown in Fig 6 The orientation of the TCP remains constant along the path.

The accuracy in the "worst-case" corner (1) at the maximum possible outreach is presented in Fig.7. It can be seen that the overshoot is reduced during positioning by feed-forward control (MFTC). Since the inverse dynamic model considers only rigid-body and friction dynamics, systematic errors remain. Thus, path deviations can be seen on the arm-side. These are mainly caused by joints' elasticities.

\section{Improving the path accuracy using ILC}

The cascaded feedback PID-type controller reacts to deviations, but has the disadvantage of the delayed following behavior. The feed-forward controller eliminates this delay to a large extent. However, it does not take into influences besides friction and rigid-body dynamics. Other systematic error influences are compensated by ILC in the third step. The ILC improves the controlled system behavior because it can effectively compensate iteratively repetitive disturbances.

The realization of ILC (13) is performed with a regularization parameter $h=1000$. The results show a fast convergence rate of path corrections and path accuracy. A low stationary Cartesian error is achieved after only 3 iterations for the investigated trajectory. The results of the control concepts is compared before and after the use of the ILC again for the defined "worst-case" corner (1) in Fig. 8 .

The improved arm-side accuracy as a result of the MFTC approach is further enhanced by the ILC. The path errors 

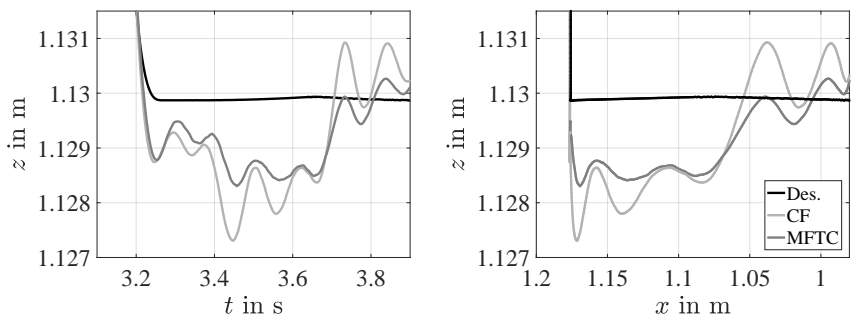

Fig. 7. Comparison of the Cartesian arm-side accuracy of the used concepts; left: time behavior in $z$-coordinate, right: behavior in $z$ - $x$-plane

resulting from joints compliance and especially the overshoot during positioning are significantly reduced. Meaningful quantitative results for the investigated trajectory (1.) and two further demanding trajectories show Tab. III. The large improvements and the robust behavior of the ILC has to be emphasized. However, some oscillations remain due to restricted sampling rate of corrections as well as simple modeling of the control loops. In the future, more sophisticated ILC approaches will be investigated.

TABLE II

PATH ERROR FOR THE USED CONCEPTS AND 3 DIFFERENT TRAJECTORIES

\begin{tabular}{|c||c|c|c||c|c|c|}
\hline \multirow{2}{*}{ No. } & \multicolumn{5}{c|}{$\varepsilon_{\mathrm{RMS}}\left(\varepsilon_{\mathrm{MAX}}\right)$ in mm } \\
& $\mathrm{x}$ & $\mathrm{y}$ & $\mathrm{z}$ & $\mathrm{x}$ & $\mathrm{y}$ & $\mathrm{z}$ \\
\hline 1. & $0.6(2.4)$ & $0.9(2.5)$ & $0.5(1.7)$ & $\mathbf{0 . 2 ( 0 . 9 )}$ & $\mathbf{0 . 4}(\mathbf{1 . 5})$ & $\mathbf{0 . 3 ( 1 . 1 )}$ \\
2. & $0.6(2.3)$ & $0.8(1.8)$ & $1.2(3.3)$ & $\mathbf{0 . 2 ( 0 . 9 )}$ & $\mathbf{0 . 3}(\mathbf{1 . 1})$ & $\mathbf{0 . 3 ( 1 . 0 )}$ \\
3. & $0.8 /(2.9)$ & $0.8(3.3)$ & $1.6(3.6)$ & $\mathbf{0 . 3}(\mathbf{1 . 7})$ & $\mathbf{0 . 4}(\mathbf{2 . 0})$ & $\mathbf{0 . 4}(\mathbf{1 . 7})$ \\
\hline
\end{tabular}

\section{CONCLUSION}

This paper presents a hierarchical concept for increasing the path accuracy of industrial robots for fast path applications. The necessary steps are discussed and evaluated by experiments under "worst-case" excitation of oscillations by maximum speed and payload.

Based on kinematic calibration as well as dynamic modeling and identification, a sufficiently accurate model of the inverse robot dynamics, suitable for real-time applications, is implemented. This is robustly parameterized using a statistical approach for experimental parameter identification. Furthermore an iterative learning controller (ILC) based on external camera measurements is designed. This can iteratively compensate unknown systematic disturbances, such joint elasticities, by corrections of the desired trajectory.

The use of the identified model in a torque control already shows a reduction of path errors. The subsequent use of ILC yields a significant improvement in arm-side path accuracy. The synergy between the different methods and their respective advantages is central for successful implementation.

For further improvements, remaining arm-side oscillations have to be considered in the ILC algorithm. Therefore, it is planned to utilize the configuration dependent eigenfrequencies of the axes' control loops within ILC models.
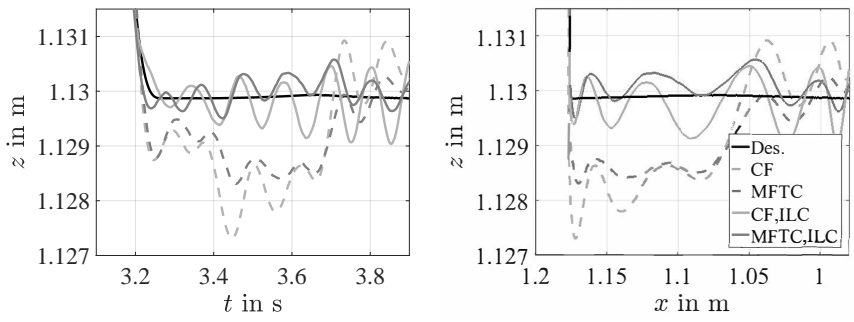

Fig. 8. Comparison of the Cartesian arm-side accuracy of the used concepts with (-) and without (- - -) ILC; left: time behavior in $z$-coordinate, right: behavior in $z$ - $x$-plane

\section{REFERENCES}

[1] W. Khalil and F. Dombre, Modeling, Identification and Control of Robots, Taylor and Francis Group, 3rd Ed., Bristol, USA, 2002.

[2] J. Swevers, C. Ganseman, D. Tükel, J. de Schutter and H. V. Brussel, Optimal Robot Excitation and Identification, IEEE, Trans. on Robotics and Automation, vol. 13(5), 1997.

[3] P. Stückelmaier, M. Grotjahn and C. Fräger, Identification of the Inverse Dynamics of a Serial Robot for Robot Drive Control, ETG-Fachbericht 146: IKMT 2015 (Köln), VDE Verlag GmbH, Berlin, Offenbach.

[4] D. Kostic et al., Modeling and Identification for High-Performance Robot Control: An RRR-Robotic Arm Case Study, IEEE, Trans. on Control Systems Technology, vol. 12(6), 2004.

[5] B. Bona and A. Curatella, Identification of Industrial Robot Parameters for Advanced Model-Based Controllers Design, IEEE, Int. Conf. on Robotics and Automation, Barcelona, 2005.

[6] K. Park, Fourier-based optimal excitation trajectories for the dynamic identification of robots, Robotica, vol. 24(5), 2006.

[7] H. Abdellatif and B. Heimann, Advanced Model-Based Control of a 6DOF Hexapod Robot, IEEE/ASME Tran. on Mech., Vol. 15, No. 2, 2010.

[8] R. W. Longman, Iterative learning control and repetitive learning control for enginneering practice, In: International Journal of Control, vol. 73, no. 10, pp. 930-954, 2000.

[9] M. Norrlöf, Iterative Learning Control: Analysis, Design, and Experiments, Nr. 653 in Linköping Studies in Science and Technology. Dissertations, Linköpings universitet, 2000.

[10] W. Hakvoort, Iterative learning control for LTV systems with applications to an industrial robot, Enschede, University of Twente, 2009.

[11] J. Wallén, Estimation-based iterative learning control, In: Linköping Studies in Science and Technology. Dissertations. No. 1358, 2011.

[12] F. Lange and G. Hirzinger, Learning to Improve the Path Accuracy of Position Controlled Robots, Proc. of the Conf. on Intelligent Robots and Systems, S. 494501, München, 1994.

[13] F. Lange and G. Hirzinger, Learning of a Controller for Non-Recurring Fast Movements, Advanced Robotics, 10(2) : 229244, 1996.

[14] F. Lange and G. Hirzinger, Learning Accurate Path Control of Industrial Robots with Joint Elasticity, Proc. of IEEE Int. Conf. on Robotics and Automation, S. 20842089, Detroit, 1999.

[15] M. Grotjahn, Kompensation nichtlinearer dynamischer Effekte bei seriellen und parallelen Robotern zur Erhöhung der Bahngenauigkeit, Mess-, Steuer- u. Regelungstech., Nr. 1003(8), VDI-Verlag, Düss., 2003.

[16] W. Khalil and J. F. Kleinfinger, A new Geometric Notation for open and closed-loop Robots, IEEE, Int. Conf. on Robotics and Automation, vol. 2, San Francisco, USA, 1986.

[17] M. Gautier and W. Khalil, A direct determination of minimum inertial parameters of robots, IEEE, Int. Conf. on Robotics and Automation, vol. 3, Philadelphia, USA, 1988.

[18] M. Gautier and W. Khalil, Direct Calculation of Minimum Set of Inertial Parameters of Serial Robots, IEEE, Trans. on Rob a. Aut., vol. 6(3), 1990.

[19] U. Wiest, Kinematische Kalibrierung von Industrierobotern, Karlsruhe, 2001.

[20] L. Ljung, System Identification: Theory for the User, New Jersey, 1999.

[21] O. Nelles, Nonlinear system identification: from classical approaches to neural networks and fuzzy models, Springer, Berlin, 2001.

[22] R. P. Paul and H. Zhang, Computationally Efficient Kinematics for Manipulators with Spherical Wrists Based on the Homogeneous Transformation Representation, The Int. J. of Rob. Research, Vol. 5(2), 1986. 